\title{
A!
}

This is an electronic reprint of the original article.

This reprint may differ from the original in pagination and typographic detail.

Palma, M.; Scheller, C. P.; Maradan, D.; Feshchenko, A. V.; Meschke, M.; Zumbühl, D. M. On-and-off chip cooling of a Coulomb blockade thermometer down to $2.8 \mathrm{mK}$

Published in:

Applied Physics Letters

DOI:

$10.1063 / 1.5002565$

Published: 18/12/2017

Document Version

Publisher's PDF, also known as Version of record

Please cite the original version:

Palma, M., Scheller, C. P., Maradan, D., Feshchenko, A. V., Meschke, M., \& Zumbühl, D. M. (2017). On-and-off chip cooling of a Coulomb blockade thermometer down to $2.8 \mathrm{mK}$. Applied Physics Letters, 111(25), 1-5. [253105]. https://doi.org/10.1063/1.5002565

This material is protected by copyright and other intellectual property rights, and duplication or sale of all or part of any of the repository collections is not permitted, except that material may be duplicated by you for your research use or educational purposes in electronic or print form. You must obtain permission for any other use. Electronic or print copies may not be offered, whether for sale or otherwise to anyone who is not an authorised user. 


\section{On-and-off chip cooling of a Coulomb blockade thermometer down to $2.8 \mathrm{mK}$}

M. Palma, C. P. Scheller, D. Maradan, A. V. Feshchenko, M. Meschke, and D. M. Zumbühl

Citation: Appl. Phys. Lett. 111, 253105 (2017); doi: 10.1063/1.5002565

View online: https://doi.org/10.1063/1.5002565

View Table of Contents: http://aip.scitation.org/toc/apl/111/25

Published by the American Institute of Physics

\section{Articles you may be interested in}

Magnetically cooling achieves record low temperatures in Coulomb blockade thermometers

Scilight 2017, 260001 (2017); 10.1063/1.5019571

Magnetic cooling for microkelvin nanoelectronics on a cryofree platform

Review of Scientific Instruments 88, 043902 (2017); 10.1063/1.4979929

Potential role of motion for enhancing maximum output energy of triboelectric nanogenerator

APL Materials 5, 074107 (2017); 10.1063/1.4979955

Chemical vapour deposition of freestanding sub- $60 \mathrm{~nm}$ graphene gyroids

Applied Physics Letters 111, 253103 (2017); 10.1063/1.4997774

Differential voltage amplification from ferroelectric negative capacitance

Applied Physics Letters 111, 253501 (2017); 10.1063/1.5006958

Enhanced recoverable energy storage density and high efficiency of $\mathrm{SrTiO}_{3}$-based lead-free ceramics

Applied Physics Letters 111, 253903 (2017); 10.1063/1.5000980

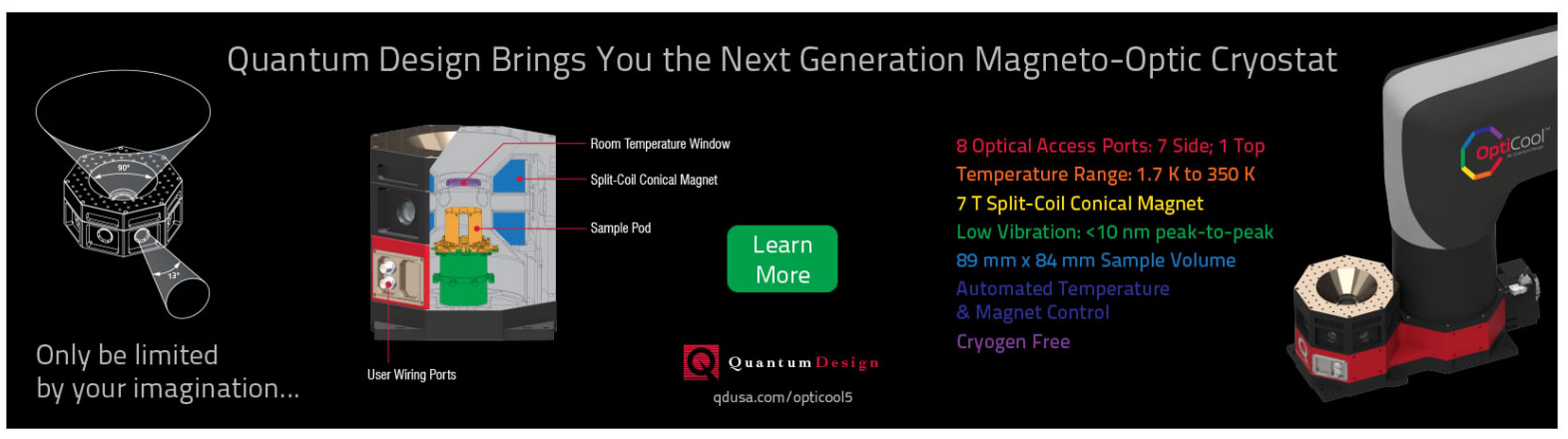




\title{
On-and-off chip cooling of a Coulomb blockade thermometer down to $2.8 \mathrm{mK}$
}

\author{
M. Palma, ${ }^{1, a)}$ C. P. Scheller, ${ }^{1, a)}$ D. Maradan, ${ }^{1,2}$ A. V. Feshchenko, ${ }^{3}$ M. Meschke, ${ }^{3}$ \\ and D. M. Zumbühl, b) \\ ${ }^{1}$ Department of Physics, University of Basel, Klingelbergstrasse 82, CH-4056 Basel, Switzerland \\ ${ }^{2}$ Physikalisch-Technische Bundesanstalt (PTB), Bundesallee 100, 38116 Braunschweig, Germany \\ ${ }^{3}$ Low Temperature Laboratory, Department of Applied Physics, Aalto University, P.O. Box 13500, \\ FI-00076 AALTO, Finland
}

(Received 30 August 2017; accepted 13 November 2017; published online 19 December 2017)

\begin{abstract}
Cooling nanoelectronic devices below $10 \mathrm{mK}$ is a great challenge since thermal conductivities become very small, thus creating a pronounced sensitivity to heat leaks. Here, we overcome these difficulties by using adiabatic demagnetization of both the electronic leads and the large metallic islands of a Coulomb blockade thermometer. This reduces the external heat leak through the leads and also provides on-chip refrigeration, together cooling the thermometer down to $2.8 \pm 0.1 \mathrm{mK}$. We present a thermal model which gives a good qualitative account and suggests that the main limitation is heating due to pulse tube vibrations. With better decoupling, temperatures below $1 \mathrm{mK}$ should be within reach, thus opening the door for $\mu \mathrm{K}$ nanoelectronics. Published by AIP Publishing.

https://doi.org/10.1063/1.5002565
\end{abstract}

Reaching ultralow temperatures in electronic transport experiments can be key to novel quantum states of matter such as helical nuclear spin phases, ${ }^{1-3}$ full nuclear spin polarization, ${ }^{4}$ quantum Hall ferromagnets, ${ }^{4}$ or fragile fractional quantum Hall states. ${ }^{5,6}$ In addition, the coherence of semiconductor and superconducting qubits ${ }^{7-9}$ as well as hybrid Majorana devices ${ }^{10-13}$ could benefit from lower temperatures. With this motivation in mind, we built a parallel network of nuclear refrigerators ${ }^{14}$ to adapt the very well established technique of Adiabatic Nuclear Demagnetization (AND) ${ }^{15-17}$ for electronic transport experiments. In this approach, the concept is to cool a nanoelectronic device directly through the electronic leads, which remain effective thermal conductors also below $1 \mathrm{mK}$. Each wire is cooled by its own, separate nuclear refrigerator in the form of a large $\mathrm{Cu}$ plate. However, despite recent progress, ${ }^{18-25}$ it remains very challenging to cool nanostructures even below $10 \mathrm{mK}$. Due to reduced thermal coupling, these samples are extremely susceptible to heat leaks such as vibrations, ${ }^{25}$ microwave radiation, ${ }^{26,27}$ heat release, ${ }^{17}$ and electronic noise. ${ }^{20}$

Metallic Coulomb blockade thermometers (CBTs) have been established as precise and reliable electronic thermometers, ${ }^{18,28,29}$ operating down to $10 \mathrm{mK}$ and slightly below. $^{21,22,24,30}$ These typically consist of linear arrays of $\mathrm{Al} /$ $\mathrm{AlO}_{\mathrm{X}} / \mathrm{Al}$ tunnel junctions with metallic islands in-between, consisting mainly of copper, see Fig. 1. The array divides the electronic noise per island by the number of junctions in series. This makes them less susceptible to electronic noise, but thermal conduction via Wiedemann-Franz cooling is not very effective through a series of resistive tunnel junctions. For this reason, the islands were enlarged into giant cooling fins ${ }^{29}$ providing a huge volume for effective electron-phonon coupling and cooling through the substrate. At low temperatures, however, this eventually fails due to the very strong $T^{5}$ temperature dependence of the electron phonon coupling.

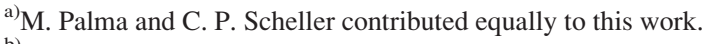

b)dominik.zumbuhl@unibas.ch.
}

On-chip nuclear demagnetization of the large fins offers itself as an elegant solution for a CBT array, providing local, in-situ cooling without having to go through potentially ineffective tunnel barriers or an insulating substrate. The large volume of the metallic islands now is taken advantage of as the spin reservoir of an AND refrigerator. At sufficiently low temperature, the electron-phonon coupling becomes so weak that the islands decouple thermally from the substrate, thus giving a low heat leak, as desired for efficient cooling. Previously, AND was applied to AlMn based single electron transistors $^{31}$ above $100 \mathrm{mK}$ and to CBTs with electroplated islands ${ }^{24}$ down to $4.5 \mathrm{mK}$ after AND. In both cases, the reduction of the electronic temperature was roughly a factor of two and the cold time a few minutes. In a home-built wet dilution refrigerator with the base temperature below $2 \mathrm{mK}$,
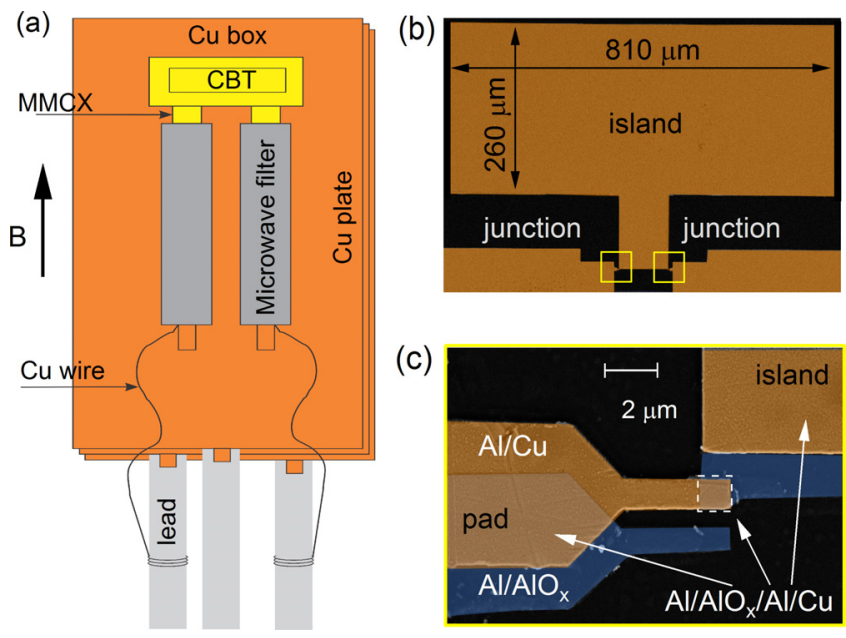

(c)

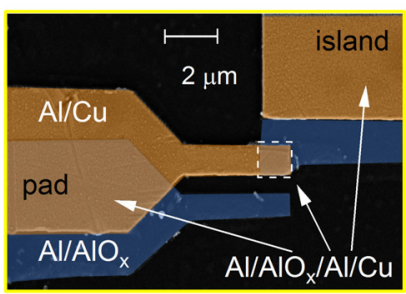

FIG. 1. (a) Schematic with CBT enclosed in a copper box (yellow), connected to Ag-epoxy microwave filters (grey), and glued onto a $\mathrm{Cu}$ plate (orange) with Ag-epoxy. (b) Electron micrograph (false color) of the CBT island (volume $\approx 42000 \mu \mathrm{m}^{3}$ ), with tunnel junctions (inside yellow rectangles) to adjacent $\mathrm{Al} / \mathrm{AlO}_{\mathrm{x}} / \mathrm{Al} / \mathrm{Cu}$ pads. (c) Zoom-in of a tunnel junction from (b), showing the overlap (white rectangle) between the top layer $(\mathrm{Al} / \mathrm{Cu}$, brown) and the bottom layer (Al/AlOx, blue). 
an electronic temperature of $3.8 \mathrm{mK}$ was reported ${ }^{22}$ without AND but with a CBT placed in a ${ }^{3} \mathrm{He}$ cell.

In this Letter, we perform AND in both the $\mathrm{Cu}$ plates in the leads and the massive CBT islands, thus combining direct on-chip cooling with a reduced external heat leak emanating from the leads. We obtain significantly improved cooling, lowering the electronic temperature by a factor of 8.6 , from $\sim 24 \mathrm{mK}$ down to $2.8 \pm 0.1 \mathrm{mK}$ with much longer cold times of a few hours. We present a simple model giving a qualitative account of the cooling cycle. The performance and in particular the precooling temperature are limited by a heat leak caused by the pulse tube vibrations of the cryo-free dilution refrigerator. With improved decoupling, the microKelvin regime in nanoelectronics should be within reach.

The present experiment is performed on a cryo-free platform, ${ }^{25,32}$ where each of the 16 leads is equipped with its own nuclear refrigerator (see Fig. 1), consisting of two moles of copper. The system allows for cooling of the $\mathrm{Cu}$ pieces down to $T_{\mathrm{Cu}}=150 \mu \mathrm{K},{ }^{25}$ see supplementary material for details. To integrate the CBT, it is placed inside a $\mathrm{Cu}$ box, see Fig. 1(a), which will also be demagnetized and further shields the CBT from high frequency radiation, arising, e.g., from higher temperature stages of the refrigerator. The $\mathrm{Cu}$ box is equipped with coaxial MMCX connectors and connected directly to additional Ag-epoxy microwave filters. ${ }^{30}$ The microwave filters used here are made from rather thick copper wire $(0.35 \mathrm{~mm}$ instead of $0.1 \mathrm{~mm})$ and contain a thicker and longer copper core, thus facilitating direct demagnetization of the filters themselves. The $\mathrm{Cu}$ box and the microwave filters are glued onto a $\mathrm{Cu}$ nuclear refrigerator with conductive Ag-epoxy in order to ensure good thermalization. The CBT consists of an array of 16 large metallic islands with $1 \mu \mathrm{m}^{2} \quad \mathrm{AlO}_{\mathrm{x}}$ tunnel barriers in-between, as shown in Figs. 1(b) and 1(c). This gives a device resistance of $150 \mathrm{k} \Omega$ and provides a rather small charging energy of $E_{c} \approx 6.5 \mathrm{mK}$, thus allowing accurate thermometry down to $\approx 2 \mathrm{mK} .{ }^{33}$ The islands are of almost macroscopic size $(\mathrm{Cu}$ layer $\approx 810 \cdot 260 \cdot 0.2 \mu \mathrm{m}^{3}$ ), making available a large reservoir of nuclear spins for demagnetization.

In order to obtain the electronic temperature of the device, we measure the 2-wire differential conductance $g$ of the CBT as a function of the bias voltage $V_{\text {bias }}$ by means of a standard low-frequency lock-in technique using a few $\mu V$ of AC excitation. Figure 2(a) shows typical conductance traces, measured at various refrigerator temperatures, as indicated. Due to Coulomb blockade effects, ${ }^{34}$ the zero bias conductance $g_{0}$ is suppressed below its asymptotic, large bias value $g_{\mathrm{T}}$. Both the width and the depth of the conductance dip are commonly used for thermometry. ${ }^{18,29}$ While fitting the full conductance trace allows one to use the CBT as a primary thermometer, using the depth of the zero bias dip requires pre-calibration and thus can be used only as a secondary thermometer. However, the primary mode is prone to overheating due to the large applied DC bias. This effect becomes particularly important at the lowest temperatures, illustrated in Fig. 2(a). The conductance $g_{0}$ measured while permanently remaining at zero bias ${ }^{35}$ is clearly lower in conductance (dark blue marker) - and thus also lower in temperature-than the one obtained from a bias sweep at the same refrigerator temperature (dark blue trace), also see
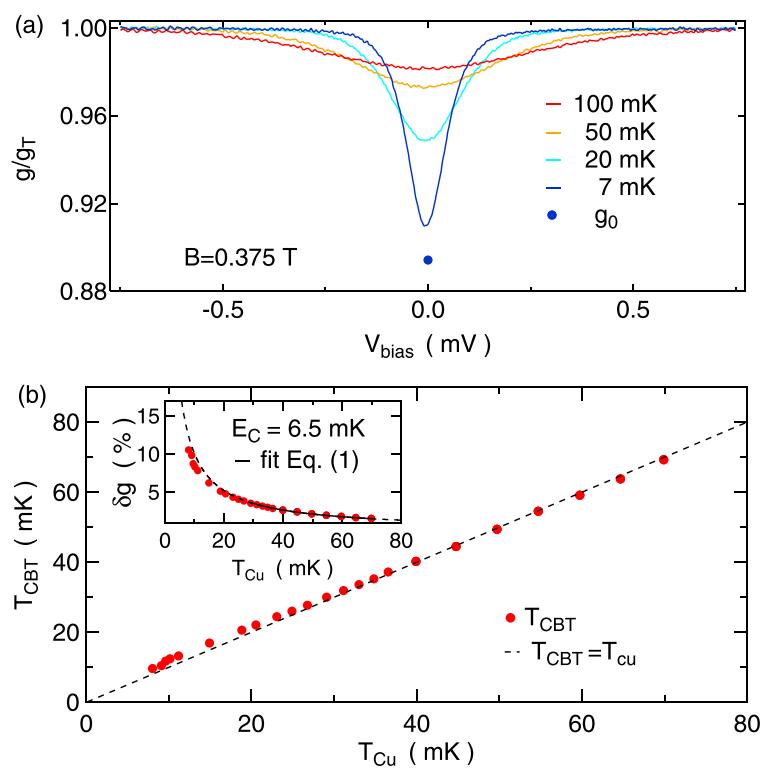

FIG. 2. (a) Bias dependence of the differential conductance $g$ normalized with the high bias conductance $g_{T}$, shown for various $\mathrm{Cu}$ plate temperatures $T_{\mathrm{Cu}}$. An in-plane field $B=0.375 \mathrm{~T}$ drives the $\mathrm{Al}$ thin films normal. The zero bias conductance (dark blue circle, measured after equilibrating at $T_{\mathrm{Cu}}=7$ $\mathrm{mK}$ ) remains clearly below the bias sweep (dark blue curve). (b) CBT temperature $T_{\mathrm{CBT}}$ versus $T_{\mathrm{Cu}}$. The diagonal dashed line indicates ideal thermalization $T_{\mathrm{CBT}}=T_{\mathrm{Cu}}$. The inset shows the normalized zero bias conductance $\operatorname{dip} \delta g$ as a function of $T_{\mathrm{Cu}}$. A fit using Eq. (1) is done over the high temperature, well thermalized regime $T_{\mathrm{Cu}} \geq 30 \mathrm{mK}$ (solid black curve) and delivers the charging energy $E_{c}$ of the device as the only fit parameter. The dashed curve indicates the low temperature extension below $30 \mathrm{mK}$ of the fit with the same $E_{c}$.

supplementary material. Therefore, the CBT is used in the secondary mode here for the rest of this work. ${ }^{18,29,30}$

The normalized Coulomb blockade zero bias dip $\delta g=1$ $-g_{0} / g_{T}$ is given by the third order expansion ${ }^{29}$

$$
\delta g=u / 6-u^{2} / 60+u^{3} / 630,
$$

for sufficiently small $u$ (see below), where $u=E_{C} / k_{B} T_{\mathrm{CBT}}$, and $k_{B}$ is the Boltzmann constant. The inset of Fig. 2(b) shows the measured $\delta g$ as a function of temperature $T_{\mathrm{Cu}}$ (red circles) along with a fit (solid black) performed in the high temperature regime above $30 \mathrm{mK}$ using Eq. (1). The fit delivers the charging energy $E_{c}=6.5 \pm 0.1 \mathrm{mK}$, which now allows us to convert the measured $\delta g$ into $T_{\mathrm{CBT}}$ for the whole temperature regime, thus providing the calibration of the secondary thermometer. The fit agrees very well with the data for high temperatures, but weak overheating is seen at lower temperatures $T_{\mathrm{CBT}} \lesssim 30 \mathrm{mK}$, see Fig. 2(b), inset.

The CBT calibration curve Eq. (1) becomes more benign at the lowest temperatures, where a much larger change in $\delta g$ is required for a given change in $T_{\mathrm{CBT}}$ compared to high temperatures. Thus, a small deviation in $\delta g$ has a very small effect on the temperature reading, and the calibration curve becomes more accurate at the lowest temperatures. The validity of Eq. (1) was investigated in detail in Ref. 33, showing that an accurate temperature reading to within $\sim 10 \%$ error or less is obtained as long as $u \lesssim 3$. In practice, this means that the obtained CBT temperature, shown in Fig. 2(b) as a function of $\mathrm{Cu}$ plate temperature $T_{\mathrm{Cu}}$, would be quite precise down to roughly $2 \mathrm{mK}$. 
In the second part of this Letter, we address the simultaneous adiabatic nuclear demagnetization of the CBT and its leads. AND is a single shot technique comprising three stages. First, magnetization of the nuclear spins at a large initial magnetic field $B_{\mathrm{i}}=9 \mathrm{~T}$ is performed. Then, the actual demagnetization is carried out down to a final field $B_{\mathrm{f}}$ of 0.375 T. Finally, the warm-up stage commences, where a heat load warms up the system until the magnetization is exhausted. Here, $B_{\mathrm{f}}$ is limited by the critical in-plane magnetic field required to break the superconductivity in the $\mathrm{Al}$ thin films present in the CBT.

Upon increasing the magnetic field to $9 \mathrm{~T}$, a large amount of heat due to the magnetization of the nuclear spins has to be drained into the mixing chamber in order to polarize the nuclear spins during precooling, depicted in Fig. 3(a). The heat switches of the parallel refrigerator network are therefore driven normal to obtain a strong thermal link to the mixing chamber. While $T_{\mathrm{Cu}}$ [orange markers, Fig. 3(a)] eventually closely approaches the mixing chamber temperature $T_{\mathrm{MC}}$ (red solid curve), the CBT temperature (blue data) is increased much more during ramping of the magnetic field to $B_{\mathrm{i}}=9 \mathrm{~T}$ and saturates well above $T_{\mathrm{MC}}$, indicating a significant heat leak onto the CBT and a weak thermal link between the CBT and the $\mathrm{Cu}$ plates. After precooling for almost 3 days, we obtain $T_{\mathrm{Cu}} \approx 10 \mathrm{mK}$ and $T_{\mathrm{CBT}} \approx 24 \mathrm{mK}$, which sets the starting point for the nuclear demagnetization. ${ }^{36}$

In the second AND step, shown in Fig. 3(b), the nuclear stage is thermally decoupled from the mixing chamber (heat switches in the superconducting state) and the magnetic field is ramped down slowly to its final value $B_{\mathrm{f}}=0.375 \mathrm{~T}$. This reduces the nuclear spin temperature according to $T_{\mathrm{f}}=T_{\mathrm{i}} \cdot B_{\mathrm{f}} / B_{\mathrm{i}}$ for an ideal adiabatic process, where $T_{\mathrm{i}}$ and $T_{\mathrm{f}}$ denote the initial and final temperature, respectively. The efficiency $\xi \leq 1$ of the process can be defined as the ratio of the realized and the ideal, adiabatic temperature reduction ${ }^{14}$ $\xi=T_{\mathrm{i}} / T_{f} \cdot B_{f} / B_{i}$, where $\xi=1$ corresponds to perfect adiabaticity. The AND process for the $\mathrm{Cu}$ plates [orange markers in Fig. 3(b)] is almost ideal (orange dashed line), resulting in an efficiency $\xi \gtrsim 0.9$. A larger deviation is observed for the CBT (compare blue data and dashed blue lines), resulting in $\xi \geq 0.35$. However, despite the reduced efficiency for the CBT, we obtain a significant reduction in CBT temperature by a factor of 8.6, giving a final $T_{\mathrm{CBT}}$ of $2.8 \pm 0.1 \mathrm{mK}$. These error bars result from the uncertainty in $E_{c}$, obtained from the curve fit in the inset of Fig. 2(b). The present measurement shows significantly improved AND efficiency compared to previous works ${ }^{22,24}$ and constitutes an important step towards the $\mu \mathrm{K}$ regime.

To get more insight into the thermal coupling of the CBT to its environment, we monitor the warm-up process after AND, shown in Fig. 3(c). While $T_{\mathrm{Cu}}$ remains almost constant during the twenty hours period of time investigated here (increase by less than $50 \mu \mathrm{K}$ ), the CBT starts to warm up immediately and reaches an equilibrium value of $7.5 \mathrm{mK}$ after eight hours. Furthermore, the CBT conductance does not recover its low temperature zero bias value when returning to zero bias after performing a bias sweep of the CBT after AND (not shown). Instead, it saturates at $7.5 \mathrm{mK}$, while the $\mathrm{Cu}$ plates are still well below $1 \mathrm{mK}$. These observations indicate a rather limited spin reservoir due to the finite size
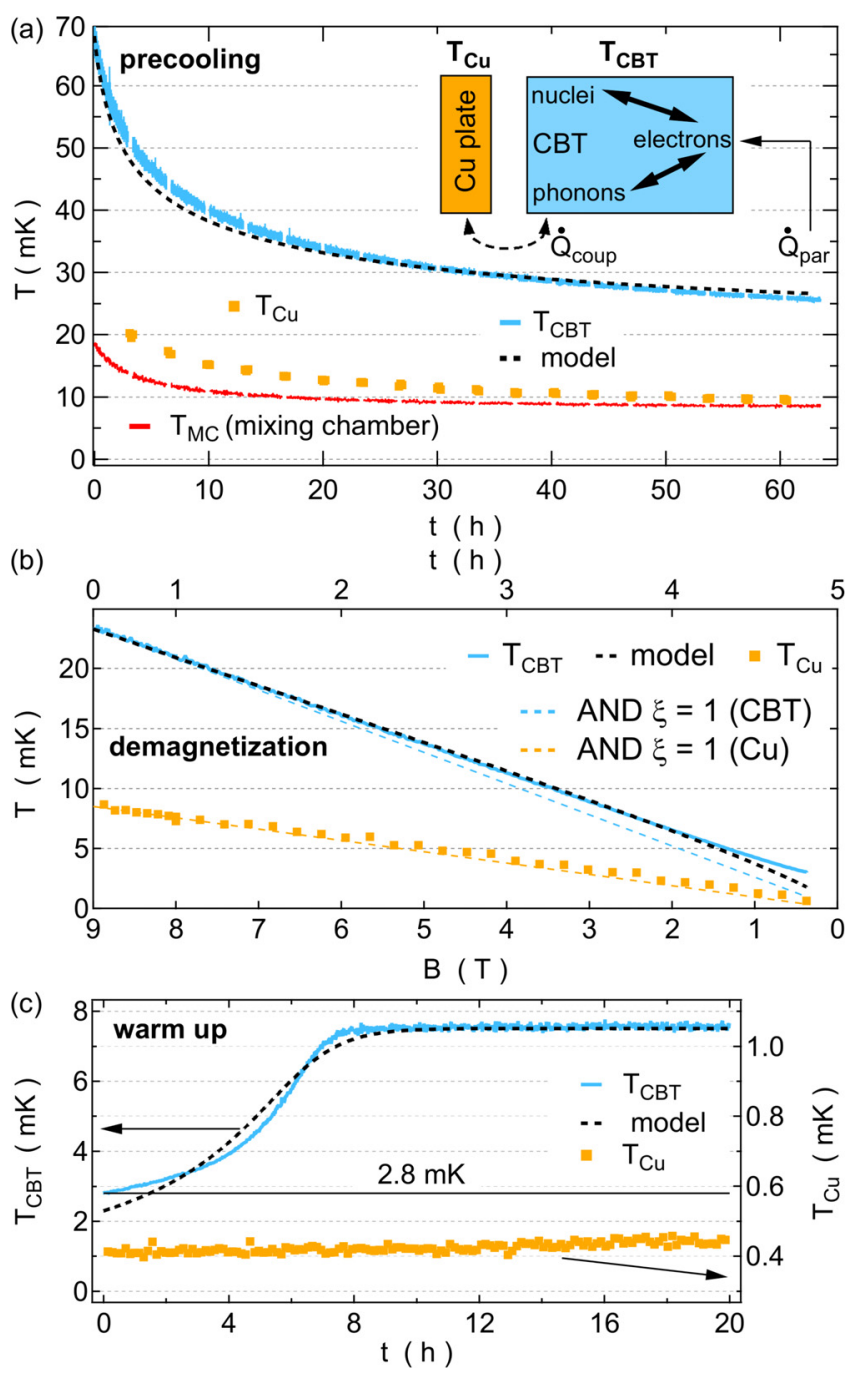

FIG. 3. (a) Various temperatures as a function of the precooling time, as labeled. The inset shows a schematic of the thermal model. (b) Evolution of various temperatures during the AND process. Blue and orange dashed lines indicate ideal cooling of $\mathrm{CBT}$ and $\mathrm{Cu}$ plates, respectively. (c) Warm-up curves for various thermometers. The model is shown as the black dashed curve for all panels.

of the CBT islands as well as a significant heat leak due to applied bias, in a limit where the CBT is well decoupled from the $\mathrm{Cu}$ plates. This indicates that the AND process directly demagnetizes the CBT, while little cooling power is provided externally from the $\mathrm{Cu}$ plates.

In order to obtain a more quantitative insight, a simple thermal model is developed [schematic in the inset of Fig. 3(a)] to capture the main features of the experiment. For the $\mathrm{Cu}$ plates and the CBT islands, we have three different thermal subsystems, namely phonons, electrons, and nuclei. We note that at low temperature, the largest contribution to the specific heat by far is provided by the nuclei. Electrons are coupled on the one hand to the nuclear bath by the hyperfine interaction via the Korringa link ${ }^{17}$ and on the other hand to the phonon bath by means of the electron-phonon interaction. Hence, nuclei and phonons are only indirectly coupled through the electronic system.

The CBT device with its large metallic islands is in principle connected thermally to the $\mathrm{Cu}$ plates through its electronic leads and through its substrate. While for a single 
tunnel junction, ${ }^{21,37}$ the two low-resistance reservoirs adjacent to the junction can be cooled efficiently through the electronic leads, Wiedemann-Franz cooling turns out to be weak for a CBT consisting of an array of resistive tunnel junctions separating the islands from the leads. Given the insulating substrate on which the CBT resides, there is only the phonon degree of freedom available for transferring heat between $\mathrm{Cu}$ plates and CBT islands, thus again giving only weak coupling. This phonon process contains several thermal resistances in series, namely, the weak electron-phonon coupling in the CBT islands (negligibly weak resistance in the $\mathrm{Cu}$ plates due to their much larger size), the acoustic mismatch at the metal-semiconductor interfaces giving rise to a Kapitza boundary resistance, ${ }^{17,38}$ and finally the weak thermal conductivity within the insulating substrate itself. In the presence of a finite heat leak onto the CBT islands, these weak thermal links lead to a significant temperature difference between $\mathrm{CBT}$ and $\mathrm{Cu}$ plates and associated long time constants, as observed, particularly during precooling.

Given such a limiting bottleneck between $\mathrm{Cu}$ plates and CBT islands, we assume the electronic, phononic, and nuclear temperatures within the CBT itself to be well coupled (bold arrows in the schematics) and equilibrated. Thus, in a simplified model, the CBT is taken as a single thermal system carrying the nuclear specific heat and is assumed to be weakly coupled to the $\mathrm{Cu}$ plate through $\dot{Q}_{\text {coup }}$ $=A\left(T_{\mathrm{CBT}}^{p}-T_{\mathrm{Cu}}^{p}\right)$ (dashed arrows), where the coupling constant $A$ and the exponent $p$ are fit parameters. Assuming a single cooling mechanism, $p=5$ corresponds to electronphonon coupling, while $p=2$ indicates Wiedemann-Franz cooling for a single island. Besides the coupling mechanism, a parasitic heat load $\dot{Q}_{\mathrm{par}}$ to the CBT is assumed to subsume, e.g., electronic noise, heat release, or pulse tube eddy current heating (see supplementary material).

We obtain qualitative agreement between the model and data for all three stages of the AND process-precool, demagnetization, and warm-up-using a single coupling constant $A=7.6 \times 10^{-12} \mathrm{~W} / \mathrm{K}^{3}$, and a static parasitic heat leak (during precool and warm-up) of $\dot{Q}_{\mathrm{par}}=32 \mathrm{aW}$ per island. However, a substantially increased dynamic heat load of $\dot{Q}_{\mathrm{par}}=485 \mathrm{aW}$ has to be assumed during demagnetization in order to explain the low demagnetization efficiency in Fig. 3(b). Furthermore, we allow for a weak temperature dependence of the coupling exponent $p$, reducing its value from $p=3.2$ during precool to $p=2.7$ during demagnetization and further down to $p=2.5$ for the warm-up process. Thus, we are seeing slightly lower exponents as temperature is decreased from precool to AND and warm-up. This is consistent with Wiedemann-Franz cooling gradually taking over from electron-phonon cooling as temperatures are lowered. Similar exponents were obtained in earlier works. ${ }^{18,22,29,30}$ Under the above assumptions, the thermal response of the CBT is qualitatively captured by the model.

We note that significant temperature differences between electronic and nuclear systems would lead to initial cooling effects during the warm-up process due to the much smaller static heat leak compared to the dynamic case. This is in contrast to the measurements, thus supporting the hypothesis of equilibrated subsystems within the CBT. We also observe that the parasitic heat determined from warm- up curves of the $\mathrm{Cu}$ plates ${ }^{25}$ is similar to that obtained from the CBT model here. ${ }^{39}$ This suggests that both the cooling power and the parasitic heat leak scale with the volume and the area of copper used. This is confirmed with measurements on a different CBT (see supplementary material), showing very similar demagnetization performance despite CBT islands which are about 150 times smaller in area and volume. Thus, the predominant heat leak does not couple in through the leads of the CBT (e.g., electronic noise or external electronic heat leak) but rather through the area or volume of the islands. This is consistent with pulse tube eddy current heating, microwave absorption, or material heat release. We note that FFT spectra, taken from the electronic noise across the CBT, show a frequency comb with a spacing of $1.4 \mathrm{~Hz}$, the pulse tube frequency (see supplementary material), thus suggesting an important role for pulse tube vibrations.

In conclusion, we demonstrate simultaneous on-and-off chip magnetic cooling of a CBT with an efficiency $\xi$ of $35 \%$, thereby lowering the device temperature by a factor of 8.6 from $24 \mathrm{mK}$ down to $2.8 \mathrm{mK}$. The CBT remains colder than the dilution refrigerator mixing chamber for more than 6 hours. Future improvements include improved microwave filtering, reduction of vibration induced eddy current heating due to active damping, better mounting, and rigid fixing of the support structure of the nuclear stage to the mixing chamber shield and magnet support assembly. ${ }^{40}$ This should improve the currently inefficient precooling process as well as reduce the large dynamic heat leak and thus reduce the final temperature after AND.

See supplementary material for the details on sample fabrication, the experimental setup, CBT mode of operation and thermalization at zero bias, further warmup of the CBT upon heating three $\mathrm{Cu}$ plates, consistent AND data from a second device with larger $E_{C}$ and smaller islands, and investigation of electrical noise and vibrations.

We would like to thank H. J. Barthelmess, R. Blaauwgeers, J. P. Pekola, G. Pickett, and P. Vorselman for useful input and discussions. The work shop teams of M. Steinacher and S. Martin are gratefully acknowledged for technical support. This work was supported by the Swiss NSF, NCCR QSIT, the Swiss Nanoscience Institute, the European Microkelvin Platform (EMP), an ERC starting grant (DMZ), and EU-FP7 MICROKELVIN and ITN Q-NET 264034 (AVF).

${ }^{1}$ P. Simon and D. Loss, Phys. Rev. Lett. 98, 156401 (2007).

${ }^{2}$ P. Simon, B. Braunecker, and D. Loss, Phys. Rev. B 77, 045108 (2008).

${ }^{3}$ C. P. Scheller, T.-M. Liu, G. Barak, A. Yacoby, L. N. Pfeiffer, K. W. West, and D. M. Zumbühl, Phys. Rev. Lett. 112, 066801 (2014).

${ }^{4}$ S. Chesi and D. Loss, Phys. Rev. Lett. 101, 146803 (2008).

${ }^{5}$ W. Pan, K. W. Baldwin, K. W. West, L. N. Pfeiffer, and D. C. Tsui, Phys. Rev. B 91, 041301 (2015).

${ }^{6}$ N. Samkharadze, I. Arnold, L. N. Pfeiffer, K. W. West, and G. A. Csáthy, Phys. Rev. B 91, 081109 (2015).

${ }^{7}$ R. Hanson, L. P. Kouwenhoven, J. R. Petta, S. Tarucha, and L. M. K. Vandersypen, Rev. Mod. Phys. 79, 1217 (2007).

${ }^{8}$ J. Clarke and F. K. Wilhelm, Nature 453, 1031 (2008).

${ }^{9}$ M. H. Devoret and R. J. Schoelkopf, Science 339, 1169 (2013).

${ }^{10}$ R. M. Lutchyn, J. D. Sau, and S. Das Sarma, Phys. Rev. Lett. 105, 077001 (2010). 
${ }^{11}$ Y. Oreg, G. Refael, and F. von Oppen, Phys. Rev. Lett. 105, 177002 (2010). ${ }^{12}$ J. Alicea, Phys. Rev. B 81, 125318 (2010).

${ }^{13}$ V. Mourik, K. Zuo, S. M. Frolov, S. R. Plissard, E. P. A. M. Bakkers, and L. P. Kouwenhoven, Science 336, 1003 (2012).

${ }^{14}$ A. C. Clark, K. K. Schwarzwälder, T. Bandi, D. Maradan, and D. M. Zumbühl, Rev. Sci. Instrum. 81, 103904 (2010).

${ }^{15}$ G. R. Pickett, Rep. Prog. Phys. 51, 1295 (1988).

${ }^{16} \mathrm{G}$. Pickett, Phys. B: Condens. Matter 280, 467 (2000).

${ }^{17} \mathrm{~F}$. Pobell, Matter and Methods at Low Temperatures (Springer, Berlin, 2007).

${ }^{18}$ L. Casparis, M. Meschke, D. Maradan, A. C. Clark, C. P. Scheller, K. K. Schwarzwälder, J. P. Pekola, and D. M. Zumbühl, Rev. Sci. Instrum. 83, 083903 (2012).

${ }^{19}$ G. Batey, A. Casey, M. N. Cuthbert, A. J. Matthews, J. Saunders, and A. Shibahara, New J. Phys. 15, 113034 (2013).

${ }^{20}$ D. Maradan, L. Casparis, T. M. Liu, D. E. F. Biesinger, C. P. Scheller, D. M. Zumbühl, J. Zimmerman, and A. C. Gossard, J. Low Temp. Phys. 175, 784 (2014)

${ }^{21}$ A. V. Feshchenko, L. Casparis, I. M. Khaymovich, D. Maradan, O.-P. Saira, M. Palma, M. Meschke, J. P. Pekola, and D. M. Zumbühl, Phys. Rev. Appl. 4, 034001 (2015).

${ }^{22}$ D. I. Bradley, R. E. George, D. Gunnarsson, R. P. Haley, H. Heikkinen, Y. A. Pashkin, J. Penttilä, J. R. Prance, M. Prunnila, L. Roschier, and M. Sarsby, Nat. Commun. 7, 10455 (2016).

${ }^{23}$ Z. Iftikhar, A. Anthore, S. Jezouin, F. D. Parmentier, Y. Jin, A. Cavanna, A. Ouerghi, U. Gennser, and F. Pierre, Nat. Commun. 7, 12908 (2016).

${ }^{24}$ D. I. Bradley, A. M. Guenault, D. Gunnarsson, R. P. Haley, S. Holt, A. T. Jones, Y. A. Pashkin, J. Penttilä, J. R. Prance, M. Prunnila, and L. Roschier, Sci. Rep. 7, 45566 (2017).

${ }^{25}$ M. Palma, D. Maradan, L. Casparis, T.-M. Liu, F. N. M. Froning, and D. M. Zumbühl, Rev. Sci. Instrum. 88, 043902 (2017).
${ }^{26}$ O.-P. Saira, A. Kemppinen, V. F. Maisi, and J. P. Pekola, Phys. Rev. B 85, 012504 (2012).

${ }^{27}$ A. B. Zorin, Rev. Sci. Instrum. 66, 4296 (1995).

${ }^{28}$ J. P. Pekola, K. P. Hirvi, J. P. Kauppinen, and M. A. Paalanen, Phys. Rev. Lett. 73, 2903 (1994).

${ }^{29}$ M. Meschke, J. P. Pekola, F. Gay, R. E. Rapp, and H. Godfrin, J. Low Temp. Phys. 134, 1119 (2004).

${ }^{30}$ C. P. Scheller, S. Heizmann, K. Bedner, D. Giss, M. Meschke, D. M. Zumbühl, J. D. Zimmerman, and A. C. Gossard, Appl. Phys. Lett. 104, 211106 (2014).

${ }^{31}$ C. Ciccarelli, R. P. Campion, B. L. Gallagher, and A. J. Ferguson, Appl. Phys. Lett. 108, 053103 (2016).

${ }^{32}$ BlueFors Cryogenics Oy Ltd., Helsinki, Finland.

${ }^{33}$ A. V. Feshchenko, M. Meschke, D. Gunnarsson, M. Prunnila, L. Roschier, J. S. Penttilä, and J. P. Pekola, J. Low Temp. Phys. 173, 36 (2013).

${ }^{34} \mathrm{G}$.-L. Ingold and Y. V. Nazarov, "Charge tunneling rates in ultrasmall junctions," in Single Charge Tunneling, NATO ASI Series B, Vol. 294, edited by H. Grabert and M. H. Devoret (Plenum, 1992), pp. 21-107.

${ }^{35}$ This requires careful input voltage drift stabilization as provided by our home built IV converter, particularly below $10 \mathrm{mK}$. Basel Electronics Lab, Physics Departement, University of Basel, Switzerland.

${ }^{36}$ This corresponds to a nuclear polarization of $\approx 40 \%$ in the $\mathrm{Cu}$ plates and $\approx 17 \%$ in the CBT islands. ${ }^{17}$

${ }^{37}$ M. Nahum and J. M. Martinis, Appl. Phys. Lett. 63, 3075 (1993).

${ }^{38}$ E. T. Swartz and R. O. Pohl, Rev. Mod. Phys. 61, 605 (1989).

${ }^{39}$ Static heat leak: $32 \mathrm{aW}$ per CBT junction, corresponding to $5.4 \mathrm{nW} / \mathrm{mol}$ $\mathrm{Cu}$, compared to typically $1-2 \mathrm{nW} / \mathrm{mol} \mathrm{Cu}$ for the large plates. Dynamic heat leak: $485 \mathrm{aW}$ per junction during AND, corresponding to $82 \mathrm{nW} / \mathrm{mol}$ $\mathrm{Cu}$, compared to an estimated $30 \mathrm{nW} / \mathrm{mol} \mathrm{Cu}$ for the large plates. ${ }^{25}$

${ }^{40}$ I. Todoshchenko, J.-P. Kaikkonen, R. Blaauwgeers, P. J. Hakonen, and A. Savin, Rev. Sci. Instrum. 85, 085106 (2014). 